\title{
Cardiac signs for students: the wheat and the chaff
}

\author{
JAMES K FINLAYSON, ALASDAIR C F KENMURE, DAVID S SHORT
}

British Medical fournal, 1978, 1, 1471-1473

Cardiology has undergone some of the greatest transitions of any branch of medicine in recent years. Not only has technological improvement increased the therapeutic possibilities but the pattern of disease has also changed greatly since the beginning of the century. Nevertheless, the teaching of clinical cardiology has remained remarkably static through the years, and students are still often encumbered with unreliable methods and outdated physical signs that have little relevance to most of the patients they will encounter in practice. Moreover, there is a conspicuous lack of uniformity about the teaching of physical signs and their meaning not only among different medical schools but even among different clinics in the same hospital. There is an urgent need to re-examine our teaching methods and reach agreement about what should and should not be taught.

The following proposals are presented as a basis for discussion. They are founded on our combined experience of over 60 years of teaching and examining students, and they have been tested by the discipline of a weekly consultative clinic at which we compare our findings after examining the patients and before we know the results of the investigations.

\section{The arterial pulse}

RATE

Great precision is rarely necessary, so if the pulse is regular it is usually sufficient to count it for six seconds and multiply by 10 . If it is irregular it should be counted for 30 seconds and doubled.

\section{RHYTHM}

If there is any doubt about the rhythm auscultation of the heart is more informative than palpation of the pulse.

\section{VOLUME AND CHARACTER}

The carotid artery is generally the best guide to the character of the pulse in the proximal aorta and provides the best indication of any abnormality caused by disease affecting the left ventricle. Palpation of the right (or failing this the left) carotid artery should therefore supersede evaluation of the pulse in the radial and brachial arteries except when simply determining rate and rhythm. The volume of the brachial and radial pulses varies greatly depending on the degree of

Cardiac Department, Aberdeen Royal Infirmary, Aberdeen JAMES K FINLAYSON, BSC, FRCP, consultant physician ALASDAIR C F KENMURE, MD, FRCP, consultant cardiologist DAVID S SHORT, PHD, FRCP, consultant physician development of the forearm muscles (compare, for example, the pulses in the arms of an office worker with those in the arms of a road sweeper), whereas the volume of the carotid pulse is much less variable. Familiarity with palpation of the carotid pulse is in any case essential for the timing of murmurs. Palpation of the femoral pulse is, of course, essential for detecting coarctation of the aorta.

The volume of the pulse is assessed by the degree of displacement of the palpating thumb. (It is quite in order to use the thumb in palpating a large artery.) It is therefore a measure of the pulse pressure - the difference between the systolic and diastolic pressures at that point.

The character of the pulse is essentially an evaluation of its upstroke, whether sharp or blunt.

There are four common abnormalities of pulse volume and character: (a) the large volume and sharp upstroke pulse seen characteristically in aortic regurgitation. Similar pulses are seen in patients with a high output state or a rigid aorta; $(b)$ the large volume and blunt upstroke pulse seen in patients with a combination of aortic stenosis and regurgitation; $(c)$ the small volume and blunt upstroke pulse seen characteristically in aortic stenosis; and $(d)$ the small volume and sharp upstroke pulse seen characteristically in mitral regurgitation. These pulse abnormalities are, of course, found only when the valve lesion is at least moderately severe.

Superfluous terms include dicrotic, anacrotic, bisferiens, water hammer. These abnormalities are difficult to recognise, unreliable, and contribute nothing to diagnosis. Pulsus paradoxus almost comes into the same category; although this sign may occasionally help in diagnosing cardiac tamponade, it is not common and when it occurs there are usually other grounds for suspecting pericardial effusion. The manoeuvre of gripping the patient's wrist and raising his arm to determine the presence or absence of a water-hammer pulse is far less sensitive and discriminating than palpation of the carotid pulse. Evaluating the state of the radial artery wall contributes nothing of value to an assessment of the state of the circulation either locally or in other more important vascular beds.

\section{Blood pressure}

The blood pressure should normally be measured in the right arm (because this is the side where the doctor usually stands when examining the patient) and if otherwise it should be stated. The conditions under which the examination is generally undertaken are such that extreme precision is meaningless; it is sufficient for most purposes to record to the nearest $5 \mathrm{~mm} \mathrm{Hg}$. In Britain, the diastolic pressure has traditionally been recorded at the fourth phase (the muffling of the Korotkoff sounds) but there is currently a strong tide flowing in the direction of preferring the fifth phase (point of disappearance of the sounds). There are good arguments for both points, but since there are a significant number of people in whom the fifth phase is absent, we advise that the recommendation of the World Health Organisation ${ }^{1}$ be followed and that both phases be recordedfor example, $160 / 105-100$ or $160 / 105-$ ? $\mathrm{mm} \mathrm{Hg}$. Recording both phases is particularly important if the diastolic pressure lies in the borderline range $(90-110 \mathrm{~mm} \mathrm{Hg}$ ) or if the two phases differ by more than $5 \mathrm{~mm} \mathrm{Hg}$. The heart (or pulse) rate should be recorded when the blood pressure is measured, because it may indicate the degree of nervousness present at the time. The presence of a "necklace flush" -a transient erythema of the neck and upper chest-should be noted for the same reason. 


\section{Jugular venous pulse}

The jugular venous pulse can be determined by inspection in over $90 \%$ of individuals. Both the pressure and the form of the pulse wave provide vital information.

Pressure-The patient should be allowed to lie comfortably with his trunk inclined at an angle of $30^{\circ}$ to $45^{\circ}$ to the horizontal. It is important to look for venous pulsation before starting to palpate the neck. The site of pulsation indicates the top of the column of blood in the vein, and the vertical distance between the level of pulsation and that of the sternal angle represents the jugular venous pressure. The actual pressure at the time of examination should be recorded. If there is a large wave the highest and lowest pressures should be noted-for example, $6 / 2 \mathrm{~cm}$. The mean pressure is usually within the range of +3 to $-6 \mathrm{~cm}$. In patients who are, or have been, in heart failure venous pulsation is often best seen in the internal jugular vein. Both veins give roughly the same reading, though the pulsation in the external jugular vein tends to be damped. The internal jugular pulse is therefore preferable. (For practical advice on determining the jugular venous pulse, see Appendix.)

Wave form-The most important abnormality of jugular wave form is the systolic surge ( $v$ wave) of tricuspid regurgitation. This often provides information about the state of the tricuspid valve which cannot readily be obtained at the bedside in any other way. The presence of a large $a$ wave indicates tricuspid stenosis or right ventricular hypertension. The presence of intermittent cannon waves may be useful in differentiating extrasystoles from atrial fibrillation and complete heart block from sinus bradycardia. Other abnormalities, such as the $y$ descent of constrictive pericarditis, rarely provide information that cannot be more reliably obtained by other means.

Hepatojugular reflux-This is a useful manoeuvre for determining the level of the jugular venous pressure but it is not a reliable sign of incipient heart failure.

\section{Apex beat}

The position of the apex beat is important, because it provides the best evidence obtainable at the bedside of the size of the heart. If the apex beat cannot be identified with the patient lying flat he should be turned on to his left side. If it can be felt only in this position it may be assumed that its position will be no further to the left when the patient is lying flat. The site of the apex beat should be recorded in relation to the mid-clavicular line, the anterior axillary line, or the mid-axillary line. The mid-line is too remote a reference point; a statement that the apex beat is $x \mathrm{~cm}$ from the mid-line is valueless unless the size and shape of the chest are known.

Character-Reliably assessing the character of the apex beat demands considerable experience. There are, however, two easily recognisable abnormalities: (a) the forcible, heaving apex beat of left ventricular hypertrophy, as seen in hypertension or aortic stenosis; and $(b)$ the hyperkinetic, large volume apex beat of left ventricular dilatation, as seen in aortic regurgitation or mitral regurgitation. No attempt should be made to teach students to recognise a right ventricular apex, because this is rarely encountered except in specialised cardiac cliniss, and even then trained cardiologists often cannot recognise it. The term "tapping" apex beat causes confusion. It is not generally recognised that the tapping quality (seen characteristically in mitral stenosis) does not represent right ventricular hypertrophy but the presence of a loud and palpable first heart sound.

\section{Left parasternal impulse}

The commonest cause of a left parasternal impulse is mitral regurgitation. The palpable impulse is caused by forward displacement of the ventricles as the left atrium is distended by the regurgitant flow. An abnormal impulse due to a hyperdynamic or hypertrophic right ventricle is much less common in ordinary clinical practice. Differentiating between the parasternal impulse of mitral regurgitation and that of a right ventricular abnormality requires exceptional experience.

\section{Auscultation}

There are four cardinal areas of auscultation; the aortic area over the second right costal cartilage, the pulmonary area in the second left intercostal space, the mitral area over the apex beat, and the tricuspid area in the fourth left intercostal space near the sternal edge.

The diaphragm should be used when auscultating all areas. The bell should be used at the mitral and tricuspid areas for detecting low frequency sounds and murmurs, but is rarely of value elsewhere.

Auscultation should be methodical: Can I hear the first sound? Can I hear the second sound? Are there more than two sounds per cardiac cycle? Is there a murmur before the first sound, after the first sound, after the second sound?

\section{Heart sounds}

The only common, readily recognisable, and important abnormalities of the heart sounds are accentuation of the first, second, third, and fourth (atrial) sounds; the opening snap of mitral stenosis; and the mid-systolic click of a prolapsed mitral valve. Few students can recognise abnormal splitting of the second sound, let alone reversed splitting. Indeed, whenever a student is certain he has heard a split second sound it nearly always proves to be an opening snap. The third heart sound can often be heard best when the patient is turned on to the left side. Soft heart sounds are usually due to hyperinflated lungs.

\section{Murmurs}

\section{SYSTOLIC}

Although the classic distinction between ejection and pansystolic murmurs should be taught, it should also be made clear that it is impossible to categorise all systolic murmurs in this way at the bedside. In severe aortic stenosis the ejection systolic murmur lengthens to resemble a pansystolic murmur. Conversely, the murmur of mitral regurgitation is not always pansystolic, but may be audible only in early, middle, or late systole. In attempting to determine the origin of a systolic murmur it is important to take into account the site of maximum intensity of the murmur, its radiation, and other evidence. A systolic murmur heard best at the aortic area nearly always originates at the aortic valve, but a systolic murmur maximal at the mitral area is by no means always mitral in origin. The murmur of aortic stenosis (as also those of tricuspid regurgitation and ventricular septal defect) may sometimes be heard best at the apex. Radiation of a murmur to the axilla favours a mitral origin, but the fact that a systolic murmur can be heard in the neck is not a reliable sign of an aortic origin.

At least as important as the site of maximum intensity are features such as the nature of any diastolic murmur, the presence or absence of atrial fibrillation, and the character of the pulse. The presence of a diastolic murmur of aortic type points to aortic valve disease and suggests that the systolic murmur is likely to be aortic in origin, though, of course, it does not prove it. Similarly, the presence of a diastolic murmur characteristic of mitral stenosis indicates the presence of mitral valve disease and suggests that the systolic murmur may be mitral in origin. The presence of atrial fibrillation suggests mitral valve disease. A blunt upstroke suggests aortic stenosis and a sharp upstroke mitral regurgitation. Nevertheless, in many cases the origin of a systolic murmur cannot be determined with certainty without the help of chest radiography and echocardiography.

\section{DIASTOLIC}

Many systolic murmurs are unimportant, but a diastolic murmur can never be ignored. The two common types of diastolic murmur can nearly always be differentiated by their timing and quality. The murmur of aortic regurgitation starts immediately after the second sound and is blowing in character. It is usually better heard at the left sternal edge than at the aortic area, and is often audible at the mitral area. It is usually best heard when the patient is sitting up, leaning forward in full expiration. The mitral diastolic murmur never starts immediately after the second sound, but is always separated from it by an interval. It is rumbling in character, and often very localised. It is usually best heard when the patient is lying on his left side. Severe aortic regurgitation may give rise to a mitral diastolic or presystolic (Austin Flint) murmur, indistinguishable from that of mitral stenosis.

\section{OTHER MURMURS}

A continuous murmur begins in systole and continues without interruption into diastole. It must be differentiated from the to and fro 
murmur of combined stenosis and regurgitation, which is characteristically silent immediately before or after the second sound.

A pericardial rub usually has a grating or squeaking quality and is often heard both in systole and diastole or superimposed on the second sound.

\section{THE EFFECT OF RESPIRATION ON MURMURS}

To detect a soft murmur, the patient should be asked to stop breathing in the phase of expiration. Inspiration often causes an appreciable increase in the intensity of systolic and diastolic murmurs originating at the tricuspid and pulmonary valves, but has no similar effect on other murmurs.

\section{Other points in physical examination}

Thrills-These are of little value and are often reported erroneously by students.

Percussion-This is of such limited value that it has no place in routine cardiac examination.

The mitral facies-This is a relic of the days before mitral stenosis could be relieved surgically, when patients developed chronic pulmonary venous congestion and fibrosis with resulting cyanosis and low cardiac output. Now the true mitral facies is rare, and dusky red cheeks are usually evidence of an outdoor occupation and cardiovascular health.

Basal crepitations-Examination of the lungs is an essential part of cardiac assessment, and may provide evidence of pleural effusion. Basal crepitations, though suggestive of pulmonary venous congestion, are also found in other conditions, and they are sometimes absent when the radiograph shows severe interstitial pulmonary oedema.

\section{Conclusion}

There are many signs other than those we have included which can be recognised with long experience and which are of undoubted value to the expert. Nevertheless, in the basic teaching of students, and in revision courses for non-cardiologists, the emphasis should be placed on those signs which are of the greatest value. To go much beyond this is likely to be counterproductive.

This paper is concerned solely with clinical signs in cardiology. It must not be forgotten that in diagnosis the history is often more important than the examination, particularly in cases of ischaemic heart disease.

\section{Appendix}

\section{PRACTICAL ADVICE ON OBSERVATION OF THE JUGULAR VENOUS} PULSE

It is important to look for venous pulsation before adjusting the patient's position or starting to palpate the neck. If venous pulsation cannot be seen with the patient lying inclined at an angle of $30^{\circ}$ to 45 to the horizontal, the following procedures should be adopted.

Firstly, an attempt should be made to trap the lower end of the external jugular veir by placing a finger lightly across the root of the neck, first on one side then on the other. If the external jugular can be made to fill by this means and if it empties rapidly when the finger is removed, it indicates that the jugular venous pressure is well within normal limits and needs no futher study.

Secondly, if this procedure fails to distend the vein the next step is to press firmly on the abdomen with the palm of one hand and watch for filling of one or both jugular veins. If venous pulsation appears with abdominal compression and subsides when the pressure is relaxed it proves that the jugular venous pressure is well within normal limits.

Thirdly, if these two manoeuvres are unsuccessful the jugular veins might be full and the top of the column of blood might be in the cranium. The patient should then be made to sit upright.
Finally, if pulsation is still not visible the jugular venous pressure is probably exceptionally low. The patient should therefore lie flat.

It is, of course, important to be sure that any pulsation that is observed is venous and not arterial. There is no difficulty if the external jugular is correspondingly distended or the level of filling varies with respiration, change of position, or abdominal compression; if the undulation is slow rather than sudden, inward rather than outward, and does not synchronise with the carotid pulse; or if there are two waves to every heart beat. Arterial pulsation can be more easily felt than seen, whereas venous pulsation can be more easily seen than felt.

In a few patients, particularly those with thick necks, it is impossible to determine the jugular venous pressure by simple inspection.

We owe a great debt to the teaching of the late Dr Paul Wood, and also to Dr Aubrey Leatham, whose book An Introduction to Examination of the Cardiovascular System ${ }^{2}$ came into our hands only after our paper had been accepted for publication. We are also grateful to Dr Byron Evans for his criticisms of the manuscript.

\section{References}

1 World Health Organisation, Arterial Hypertension and Ischaemic Heart Disease: Preventive Aspects, Technical Report Series No 231. Geneva, WHO, 1962.

${ }^{2}$ Leatham, A, Introduction to Examination of the Cardiovascular System. London, OUP, 1977.

(Accepted 27 February 1978)

\section{What anticonvulsants are least desirable during pregnancy?}

There have been numerous reports of congenital malformations, particularly cleft lip, cleft palate, and heart defects in infants born to mothers taking anticonvulsants. The drugs implicated include all the antiepileptic drugs. The evidence that these really cause an appreciably higher incidence of malformations is poor. I think that if a woman is a well-controlled epileptic and the concentrations of the drugs in her blood are at the normal therapeutic concentrations treatment need not be changed because she is pregnant. Phenytoin is probably the most efficient drug for treating epilepsy, and it should not be discontinued because of pregnancy. There might be a case for not starting phenytoin if the first epileptic attack occurs during the first trimester and it was thought desirable to treat the epilepsy at all.

The general impression from studies ${ }^{12}$ of the teratogenic potential of anticonvulsants is that there was minimal evidence that exposure to phenytoin caused appreciably higher incidence of congenital defects. The effects could not be attributed necessarily solely to medication. Other factors might have included folic acid deficiency; the frequency and severity of seizures during pregnancy; and genetic factors, such as family history of malformations. The Committee on Safety of Medicines finally concluded that the use of anticonvulsants as a whole during pregnancy carried only a very small risk of the production of malformed infants. Few authorities would recommend withholding medication.

' Ajodhia, J M, and Hope, G M, Pharmaceutical fournal, 1973, 1, 566

Shapiro, S, et al, Lancet, 1976, 1, 272.

What are the hazards of rubella immunisation?

The only major hazard of rubella vaccination is that the live attenuated virus, if administered during pregnancy, could infect the fetus and produce congenital abnormalities. This risk applies to a pregnancy conceived up to two months after vaccination. Women should therefore be warned that for at least two months after vaccination they must continue with oral contraceptives. Side effects are uncommon, although neurc!ogical symptoms have been reported, but a cause and effect relationship has not been established. 